\title{
Transarterial chemoembolization with radiofrequency ablation versus hepatectomy in hepatocellular carcinoma beyond the Milan criteria: a retrospective study
}

This article was published in the following Dove Press journal:

Cancer Management and Research

\author{
Hang Yuan* \\ Ping Cao* \\ Hai-Liang Li \\ Hong-Tao Hu \\ Chen-Yang Guo \\ Yan Zhao \\ Quan-Jun Yao \\ Xiang Geng
}

Minimally Invasive and Interventional Department, Affiliated Cancer Hospital of Zhengzhou University, Henan Cancer Hospital, Zhengzhou 450008, China

*These authors contributed equally to this work
Correspondence: Hai-Liang Li Minimally Invasive and Interventional Department, Affiliated Cancer Hospital of Zhengzhou University, Henan Cancer Hospital, No. 127, Dongming Road, Zhengzhou 450008, China

Tel +86I3838585250

Fax $+86037 \mid 6596 \quad 1505$

Email lihailianggy@।63.com
Purpose: To compare the efficacies of transarterial chemoembolization (TACE) combined with radiofrequency ablation (RFA) with hepatectomy. Prognostic factors for the patient groups were analyzed.

Patients and methods: Data of 314 newly diagnosed cases of hepatocellular carcinoma beyond the Milan criteria were studied from January 2012 to December 2013 in our hospital. Forty-four patients were excluded owing to loss to follow-up (27 cases) or missing imaging data (17 cases); finally, 270 patients were included. All patients underwent TACE combined with RFA (TR group, 136 patients) or hepatectomy (HT group, 134 patients). Efficacy evaluation and prognostic factor analysis of the groups were conducted. Overall survival (OS) rate, progression-free survival (PFS) rate, and major complications were recorded.

Results: The 1-, 2-, 3-, and 5-year OS rates and median survival times were 98.5\%, 83.1\%, $66.2 \%, 37.1 \%$, and 46 months, respectively, for the TR group and $89.6 \%, 69.4 \%, 53.7 \%$, $30.3 \%$, and 38 months, respectively, for the HT group. There were significant statistical differences in survival rate and median survival time between the groups. Median PFS was 21 months for the TR group and 8 months for the HT group. Difference between groups was statistically significant. Multivariate analysis showed treatment method, tumor diameter and number, Child-Pugh classification, antiviral therapy, and alpha-fetoprotein levels were the independent factors affecting OS rates. Treatment mode and tumor diameter and number were the independent factors affecting PFS.

Conclusion: TACE with RFA is superior to hepatectomy in hepatocellular carcinoma treatment beyond the Milan criteria.

Keywords: hepatocellular carcinoma, hepatectomy, radiofrequency ablation, transarterial chemoembolization, Milan criteria

\section{Introduction}

Hepatocellular carcinoma (HCC) is one of the most common malignancies in the world. It has a high incidence, ranking fifth, and a high mortality rate, ranking third, especially in East Asian countries. ${ }^{1-3}$ China has the world's largest incidence of HCC, accounting for $55 \%$ of global HCC population. ${ }^{4,5}$ Regarding HCC beyond the Milan criteria with larger tumor diameter or number, there is a risk of insufficient liver reserves and of liver failure after surgical resection, especially in patients with cirrhosis as their risk of postoperative liver failure is greater. Transarterial chemoembolization (TACE) is recommended for the treatment of $\mathrm{HCC}$ beyond the Milan criteria. ${ }^{6}$ However, TACE 
is a palliative treatment and often needs to be repeated. Therefore, new treatment methods are needed to improve HCC treatment outcomes.

Radiofrequency ablation (RFA) is currently the most widely used local ablation technique. Studies have shown that short- and long-term efficacies of surgical resection and RFA for small HCCs are not significantly different. RFA can also be used for tumors with diameters $>5 \mathrm{~cm}$ or for multiple tumors. ${ }^{7}$ TACE with RFA for liver cancer treatment is superior to TACE alone, ${ }^{8}$ and surgical treatment of HCC beyond the Milan criteria is better than TACE alone. ${ }^{9}$ There are only few reports, with a small number of patients, which focus on the comparison between TACE with RFA and hepatectomy for HCC beyond the Milan criteria. ${ }^{10-12}$ So, we retrospectively analyzed clinical data of TACE combined with RFA and hepatectomy in newly diagnosed HCC patients, comparing the efficacies of the two treatment methods and analyzing the prognostic factors.

\section{Patients and methods}

A retrospective analysis of the HCC patients at our hospital from January 2012 to December 2013 was conducted. The diagnosis of HCC was based on guidelines of the European Association for the Study of the Liver ${ }^{6}$ and are as follows: demonstration of typical features of $\mathrm{HCC}$ with two imaging modalities, demonstration of typical features of HCC with one imaging modality together with an alpha-fetoprotein (AFP) level $>400 \mathrm{ng} / \mathrm{mL}$, or demonstration of cytologic and/ or histologic evidence of HCC. According to the inclusion criteria, we screened 314 patients and excluded 44 patients owing to loss to follow-up (27 cases) or missing imaging data (17 cases); finally, 270 patients were included. All patients had no treatment contraindications. Patients were divided into TACE with RFA group (TR group) and hepatectomy group (HT group) according to the initial treatment. The inclusion criteria were as follows: 1) no previous treatment for HCC;2) HCC beyond the Milan criteria (Milan criteria: single nodule with size $\leq 5 \mathrm{~cm}$ or three nodules or fewer with tumor size $\leq 3 \mathrm{~cm}$ ); 3) no vascular invasion and extrahepatic metastasis; 4) liver function of Child-Pugh A-B, Eastern Cooperative Oncology Group score 0 points; and 5) no other history of malignancy and no major organ dysfunction. The study was approved by the Ethics Committee of Henan Cancer Hospital, and written informed consent was obtained from each patient.

All patients underwent preoperative and imaging examinations including electrocardiogram, chest computed tomography (CT) plain scan, abdominal ultrasound, and abdominal three-phase CT or magnetic resonance imaging (MRI). Laboratory tests included the following: full blood count, liver and kidney function tests, electrolytes, blood glucose, serum prealbumin, coagulation function, urinalysis, stool test plus fecal occult blood test, hepatitis B 5 items, hepatitis B virus (HBV)-DNA, hepatitis C antibody, blood typing, HIV antibody, syphilis antibodies, AFP, and CA19-9. Written informed consent was obtained from all patients before treatment.

\section{TACE procedure}

TACE was performed by two radiologists with $>10$ years of interventional therapy experience. ${ }^{8,13}$ Following the introduction of $5 \mathrm{~F}$ catheter (Cook Medical, Bloomington, IN, USA) through the femoral artery, superior mesenteric artery angiography and hepatic arteriography were performed to determine portal patency and intrahepatic tumor blood supply. Then, the $2.7 \mathrm{~F}$ microcatheter was placed in the tumor-feeding arteries and confirmed by angiography. Chemoembolization was performed using $50 \mathrm{mg}$ pirarubicin with 5-20 mL of lipiodol (Guerbet Laboratories, Roissy, France). Gelatin sponge particles $(350-560 \mu \mathrm{m})$ or embolism microspheres (300-500 $\mu \mathrm{m})$ were added to supplement embolism. When the contrast column cleared within two to five heartbeats, it was considered the endpoint of the embolization.

\section{RFA procedure}

RFA was guided by CT (GE Large Aperture CT; GE Healthcare Bio-Sciences Corp., Piscataway, NJ, USA) and performed within 3-7 days after the TACE treatment. RFA was performed by radiologists with $\geq 10$ years of experience in hepatic RFA. CT scanning was performed to determine the tumor number, location, and size. The puncture site and the needle route were selected. Under the guidance of repeated CT scans, ablation was performed using a progressive needle approach with RFA needle (2-4 cm; Boston Scientific, Marlborough, MA, USA). The ablation range exceeded the tumor edge by $0.5-1.0 \mathrm{~cm}$ or more. Track ablation was performed upon withdrawal of RFA electrode to prevent bleeding and tumor seeding.

\section{Hepatectomy procedure}

Hepatectomy was carried out under general anesthesia using a right subcostal incision. Perihepatic ligaments and adhesion tissue were separated, and abdominal exploration was performed. Intraoperative ultrasound was used to assess the number, size, distribution, and invasion of adjacent structures by liver tumors, and the relationship between tumors and vessels, bile ducts, and other structures. Pringle's method was used to block hepatic blood flow. A rubber tourniquet was used to fasten the hepatoduodenal ligament. According to the tumor location, size, and number, the patients underwent hepatic lobectomy, hepatic 
segmentectomy, semi-hepatic resection, or partial resection. All procedures of hepatectomy are performed according to the standard methods recommended by the guidelines. All nodules were removed and sent to the pathology department.

\section{Follow-up}

After 4-6 weeks of treatment, the results of full blood count, liver and kidney function tests, serum AFP, ultrasound, chest CT scan, and three-phase CT or MRI scan of the upper abdomen were reviewed. In the absence of active lesions, follow-up was performed every 2-3 months. Follow-up procedures were the same as above. Treated tumors were evaluated using the modified RECIST criteria, and complete response, partial response, stable disease, and progressive disease were recorded. ${ }^{14}$ Overall survival (OS) was defined from the start of patient enrollment to death or the final follow-up endpoint on December 31, 2017.

\section{Statistical analysis}

For comparison of continuous variables, Student's $t$ or rank sum test was used, and chi-squared test was used for categorical variables. OS was calculated by Kaplan-Meier method and compared by log-rank test, and survival rate and median survival time were estimated. Univariate analysis screened factors that affected prognosis; a multivariate Cox proportional hazards regression model screened for independent prognostic factors, expressed as the risk ratio and its $95 \%$ CI. Variables with $P<0.05$ in the univariate analysis were included in the multivariate analysis. All statistical analyses were performed using SPSS 22.0 statistical software; a difference was considered significant for $P<0.05$.

\section{Results}

\section{Patient characteristics}

Of the 270 patients, 221 were males and 49 were females, aged 33-85, with an average age of 54.29 \pm 10.02 years. Statistical analysis showed no significant differences between the two groups in age, sex, HBsAg, HBeAg, HBV-DNA, $\mathrm{HCV}-\mathrm{Ab}$, cirrhosis, antiviral therapy, diabetes, preoperative liver function, Child-Pugh classification, preoperative AFP levels, hemoglobin, platelets, maximum tumor diameter, total tumor diameter, tumor number, tumor distribution, and other factors $(P>0.05)$. Patient characteristics are shown in Table 1.

In the TR group, total TACE treatment was performed 518 times (average: 3.81 times/case) and total local ablations were performed 265 times (average: 1.95 times/case). There are four patients in the TR group received liver resection after combination treatment. For these four patients, while we finished the follow-up, they were still alive. But the survival time was calculated only from the initial TACE to the time of surgery, the survival time after surgery was not included.

Histopathology of the surgical group showed 105 cases of $\mathrm{R} 0$ resection and 29 cases of $\mathrm{R} 1$ resection ( $\mathrm{R} 0$ : surgery with clear histological margins; R1: surgery with positive microscopic resection margins). There were 67 cases of

Table I Baseline characteristics of patients

\begin{tabular}{|c|c|c|c|c|}
\hline Variable & TR group $(n=136)$ & HT group $(n=134)$ & $\chi^{2}(t)$ & $P$-value \\
\hline Age (years) ${ }^{a}$ & $54.74 \pm 9.90$ & $53.83 \pm 10.15$ & 0.743 & 0.458 \\
\hline $\operatorname{Sex}(M / F)$ & $109 / 27$ & $112 / 22$ & 0.536 & 0.464 \\
\hline $\mathrm{HBsAg}$ (positive/negative) & $119 / 17$ & $|13 / 2|$ & 0.561 & 0.454 \\
\hline $\mathrm{HBeAg}$ (positive/negative) & $49 / 87$ & $42 / 92$ & 0.663 & 0.415 \\
\hline HBV-DNA (positive/negative) & $96 / 40$ & $|03 / 3|$ & 1.372 & 0.241 \\
\hline HCV-Ab (positive/negative) & $7 / 129$ & $6 / 128$ & 0.066 & 0.797 \\
\hline Cirrhosis (yes/no) & $118 / 18$ & $113 / 23$ & 0.718 & 0.397 \\
\hline Antiviral (yes/no) & $104 / 32$ & $96 / 38$ & 0.820 & 0.365 \\
\hline Diabetes (yes/no) & $8 / 128$ & $11 / 123$ & 0.559 & 0.455 \\
\hline Child-Pugh classification (A/B) & $122 / 14$ & $126 / 8$ & 1.686 & 0.194 \\
\hline Preoperative AFP $(\geq 1<400 \mathrm{ng} / \mathrm{mL})$ & $68 / 68$ & $79 / 55$ & 2.182 & 0.140 \\
\hline $\operatorname{HGB}(g / L)^{a}$ & $|37.15 \pm| 4.8 \mid$ & $140.03 \pm 14.62$ & -1.507 & 0.161 \\
\hline $\operatorname{PLT}\left(\times 10^{9} / \mathrm{L}\right)^{\mathrm{a}}$ & $|4| .73 \pm 54.7$ & $152.97 \pm 59.6$ & -1.616 & 0.107 \\
\hline PT $(s)^{a}$ & $12.74 \pm 3.12$ & $12.62 \pm 2.84$ & 0.330 & 0.791 \\
\hline Maximum diameter $(\mathrm{cm})^{\mathrm{a}}$ & $7.32 \pm 2.14$ & $7.34 \pm 2.88$ & -0.897 & 0.370 \\
\hline Total diameter $(\mathrm{cm})^{\mathrm{a}}$ & $8.89 \pm 2.95$ & $8.66 \pm 3.21$ & -0.900 & 0.368 \\
\hline Tumor number $(1 / 2 / 3 / 4 / 5)$ & $87 / 26 / 11 / 8 / 4$ & $83 / 30 / 14 / 3 / 4$ & 2.998 & 0.558 \\
\hline Tumor distribution (right/left/bilobar) & $94 / 19 / 23$ & $97 / 22 / 15$ & 1.936 & 0.380 \\
\hline
\end{tabular}

Notes: Unless otherwise indicated, data are numbers of patients. ${ }^{a}$ Data are mean $\pm S D$.

Abbreviations: AFP, alpha-fetoprotein; HBeAg, hepatitis B virus e antigen; $H B s A g$, hepatitis B surface antigen; HBV-DNA, hepatitis B virus DNA; HCV-Ab, hepatitis C virus antibody; HGB, hemoglobin; HT, hepatectomy; PLT, platelet; PT, prothrombin time; TR, transarterial chemoembolization with radiofrequency ablation. 
hepatectomy, 52 cases of hepatic segmentectomy, 11 cases of semi-hepatic resection, and 4 cases of hepatic segmentectomy plus partial resection. According to review results, 39 cases $(29.10 \%)$ recurred within 3 months postoperation, 66 cases (49.25\%) recurred within 6 months, and 94 cases (70.15\%) recurred within 12 months. Thirteen patients underwent a second surgical resection. Seventy-eight patients underwent TACE treatment for a total of 290 times (average: 3.72 times/case). Seventy-three patients underwent local ablation for a total of 133 times (average: 1.82 times/ case). The difference in the number of TACE with RFA between patients of the two groups was not statistically significant $(P>0.05)$.

\section{Complications}

The main complications of TACE treatment were nausea, vomiting, fever, abdominal pain, and transient liver dysfunction. Symptomatic and hepatoprotective treatments were relieved by symptomatic supportive treatment with no serious adverse events. After RFA, hepatic abscess occurred in one case of biliary peritoneal fistula and the patient recovered after pus drainage. There was also one case each of diaphragmatic injury and hepatic failure with recovery after symptomatic treatment, one case of gastrointestinal bleeding with recovery after hemostasis, and one case of death due to irreversible post-procedure hepatic failure. The main complications after hepatectomy included fever, transient liver injury, and pleural effusion, all relieved after symptomatic supportive therapy. However, there were three cases of abdominal hemorrhage, two of hepatic failure, four of bile leakage, five of abdominal infection, and one of intestinal obstruction. All patients recovered after symptomatic treatment and three patients died in the perioperative period because of severe hepatic failure and infection. The severe complications that occurred in both groups are shown in Table 2.

\section{Survival}

The 1-, 2-, 3-, and 5-year OS rates were 98.5\%, 83.1\%, $66.2 \%$, and $37.1 \%$, respectively, in the TR group and $89.6 \%$, $69.4 \%, 53.7 \%$, and $30.3 \%$, respectively, in the HT group. The

Table 2 Severe complications of the two groups

\begin{tabular}{|l|l|l|l|l|}
\hline Groups & Yes & No & $\chi^{2}$ & $P$-value \\
\hline TR group & 5 & 131 & 8.244 & 0.004 \\
HT group & 18 & 116 & & \\
\hline
\end{tabular}

Note: Data are numbers of patients.

Abbreviations: HT, hepatectomy; TR, transarterial chemoembolization with radiofrequency ablation. average median survival times of the TR and HT groups were 46 and 38 months, respectively. The difference between the groups was statistically significant (log-rank test, $\chi^{2}=5.652$, $P=0.017)$. The survival time curve of the groups is shown in Figure 1.

The progression-free survival (PFS) rates in 3, 6, and 12 months were $97.1 \%, 89.7 \%$, and $67.6 \%$, respectively, in the TR group and $70.9 \%, 50.7 \%$, and $29.8 \%$, respectively, in the HT group. The median PFS in the TR and HT groups were 21 and 8 months, respectively, and the difference between the groups was statistically significant (log-rank test, $\chi^{2}=18.864$, $P<0.001)$. The PFS time curve of the groups is shown in Figure 2.

\section{Univariate analysis}

Univariate analysis (Kaplan-Meier method) showed that treatment methods, age, antiviral therapy, Child-Pugh classification, preoperative AFP levels, maximum tumor diameter, total tumor diameter, and tumor number were the prognostic factors (Table 3). Treatment methods, age, preoperative AFP levels, maximum tumor diameter, total tumor diameter, and tumor number were the factors that affected PFS (Table 4).

\section{Multivariate analysis}

Multivariate Cox proportional hazards regression model analysis showed that treatment methods, maximum tumor diameter, tumor number, Child-Pugh classification, antiviral therapy, and preoperative AFP levels were the independent factors that affected OS (Table 5). Treatment method, maximum tumor diameter, and tumor number were the independent factors affecting PFS (Table 6).

\section{Discussion}

Clinical application of TACE in HCC treatment is extensive. Its advantages include the following: 1) less trauma and quicker recovery; 2) possibility of simultaneous treatment of multiple lesions; and 3) chance of radical surgery or local ablation following TACE treatment. However, because of the complex nature of the HCC tumor-feeding artery, complete embolization is difficult. Tumor blood vessels easily regenerate and the portal vein is involved in tumor blood supply. Therefore, TACE alone cannot completely inactivate tumor cells, and its long-term effect is less than optimal. ${ }^{15,16}$ In this study, we used TACE with RFA for HCC treatment. This combined treatment can inactivate the whole tumor, completely kill the target lesion, and make cure possible for HCC patients who are unsuitable or unwilling to undergo 


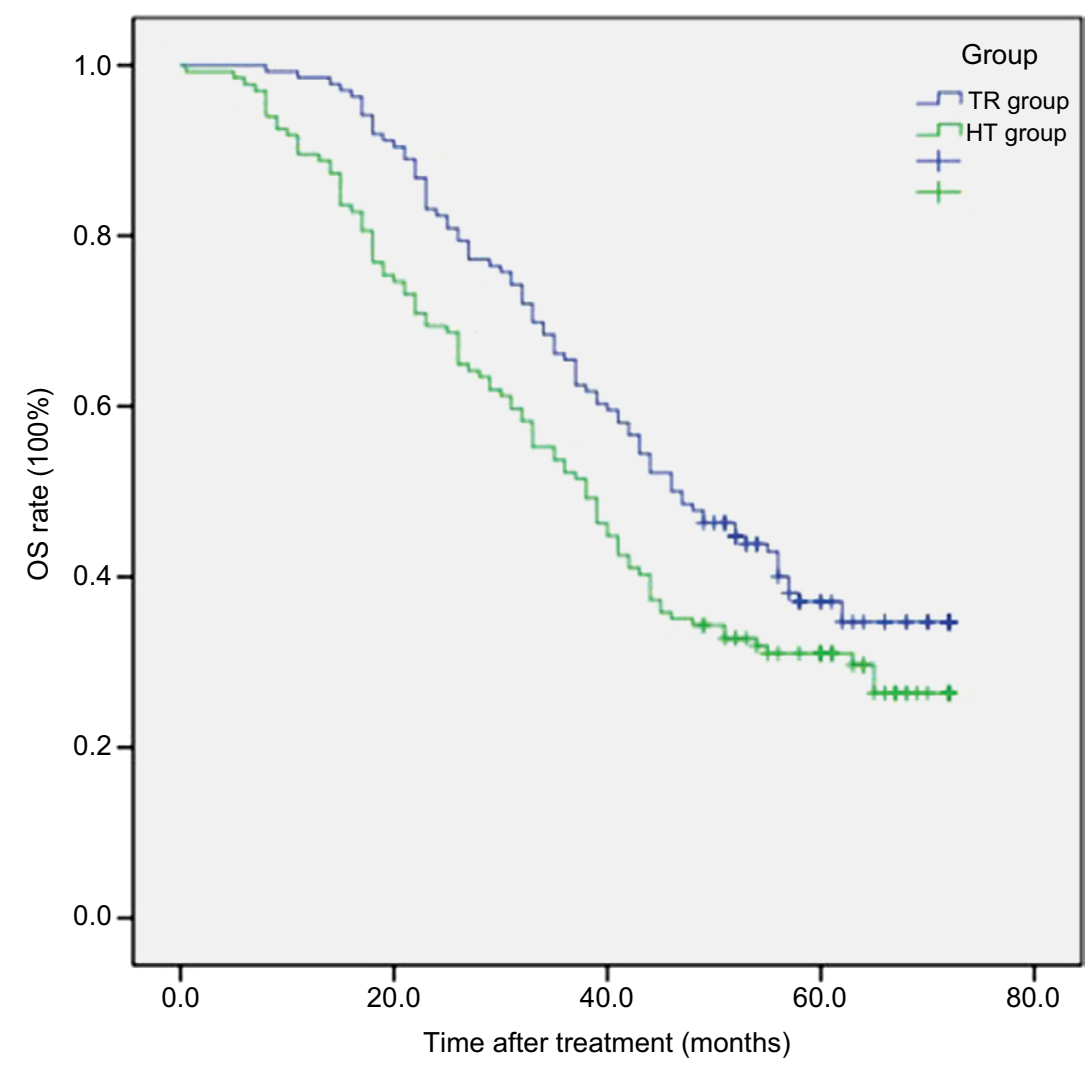

Figure I The OS of the two groups ( $P=0.017$, log-rank test).

Abbreviations: $\mathrm{HT}$, hepatectomy; OS, overall survival; TR, transarterial chemoembolization with radiofrequency ablation.

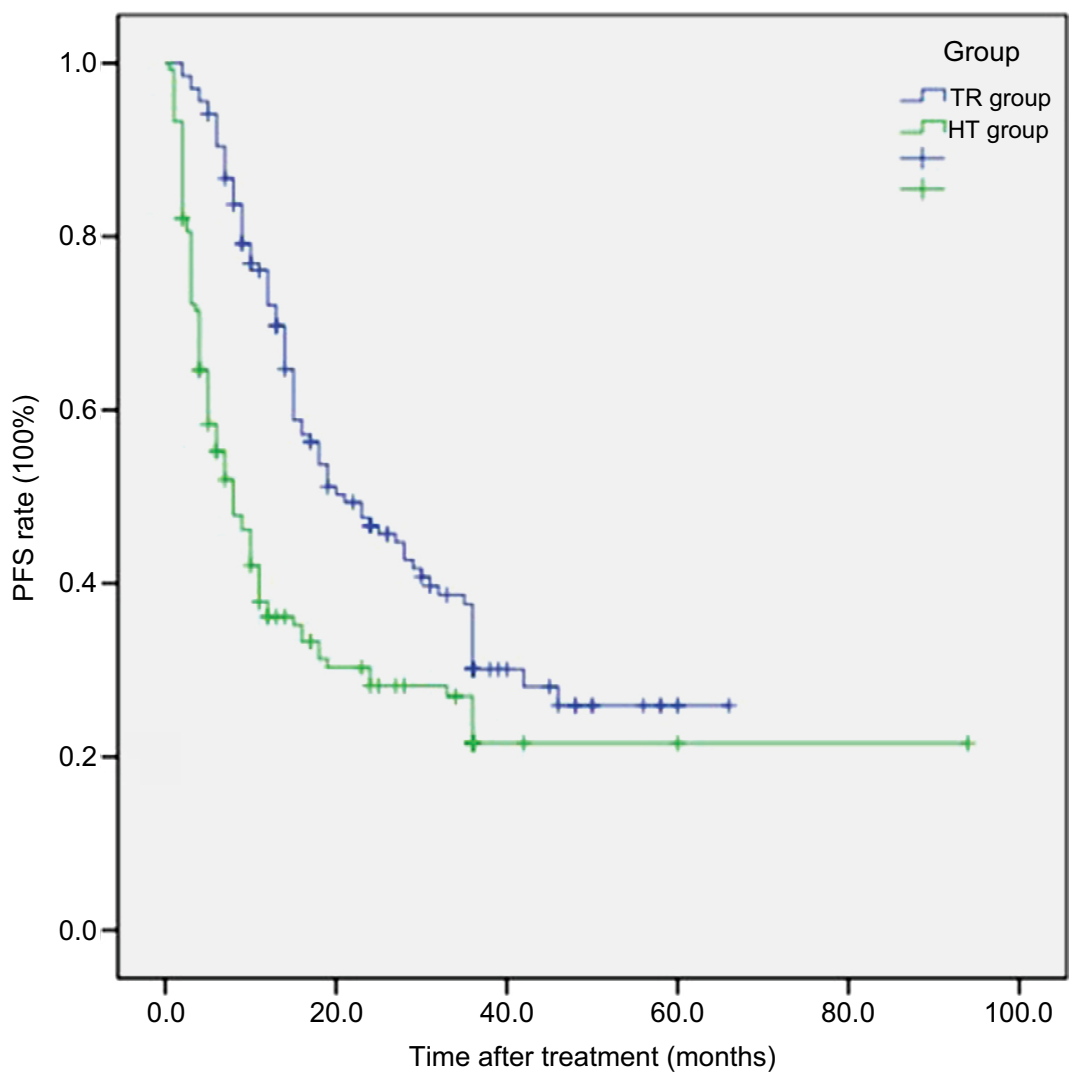

Figure 2 The PFS of the two group $(P<0.001$, log-rank test).

Abbreviations: HT, hepatectomy; PFS, progression-free survival; TR, transarterial chemoembolization with radiofrequency ablation. 
Table 3 Univariate analysis of prognostic factors of OS

\begin{tabular}{|l|l|l|l|}
\hline Variable & $\mathbf{n}$ & $\chi^{2}$ & P-value \\
\hline Treatment (TR/HT group) & $136 / 134$ & 5.652 & 0.017 \\
Sex(M/F) & $221 / 49$ & 0.022 & 0.882 \\
Age $(>/ \leq 50$ years) & $190 / 80$ & 10.825 & 0.001 \\
HBsAg (positive/negative) & $232 / 38$ & 0.020 & 0.887 \\
HCV-Ab (positive/negative) & $13 / 257$ & 0.008 & 0.927 \\
Antiviral (yes/no) & $200 / 70$ & 7.369 & 0.007 \\
Diabetes (yes/no) & $19 / 251$ & 0.090 & 0.764 \\
Child-Pugh classification $(\mathrm{A} / \mathrm{B})$ & $248 / 22$ & $4.08 \mathrm{I}$ & 0.043 \\
Preoperative AFP $(\geq /<400$ & $147 / I 23$ & 20.869 & $<0.001$ \\
ng/mL) & & & \\
Maximum diameter $(>/ \leq 7 \mathrm{~cm})$ & $113 / 157$ & 83.924 & $<0.00 \mathrm{I}$ \\
Total diameter $(>/ \leq I 0 \mathrm{~cm})$ & $63 / 207$ & 43.526 & $<0.00 \mathrm{I}$ \\
Tumor number $(>/ \leq 2)$ & $44 / 226$ & 5.676 & 0.017 \\
Tumor distribution $($ single & $232 / 38$ & 0.001 & 0.980 \\
leaf/bilobar) & & & \\
\hline
\end{tabular}

Abbreviations: AFP, alpha-fetoprotein; $\mathrm{HBsAg}$, hepatitis B surface antigen; HCV-Ab, hepatitis C virus antibody; HT, hepatectomy; OS, overall survival; TR, transarterial chemoembolization with radiofrequency ablation.

Table 4 Univariate analysis of prognostic factors of PFS

\begin{tabular}{|c|c|c|c|}
\hline Variable & $\mathbf{n}$ & $\chi^{2}$ & $P$-value \\
\hline Treatment (TR/HT group) & $136 / 134$ & 18.864 & $<0.001$ \\
\hline $\operatorname{Sex}(M / F)$ & $221 / 49$ & 0.621 & 0.431 \\
\hline Age $(>\mid \leq 50$ years $)$ & $190 / 80$ & 10.774 & 0.001 \\
\hline HBsAg (positive/negative) & $232 / 38$ & 0.007 & 0.934 \\
\hline HCV-Ab (positive/negative) & $13 / 257$ & 0.769 & 0.381 \\
\hline Antiviral (yes/no) & $200 / 70$ & 2.583 & 0.108 \\
\hline Diabetes (yes/no) & $|9 / 25|$ & 0.005 & 0.946 \\
\hline Child-Pugh classification (A/B) & $248 / 22$ & 2.493 & 0.114 \\
\hline $\begin{array}{l}\text { Preoperative AFP }(\geq 400 /<400 \\
\mathrm{ng} / \mathrm{mL})\end{array}$ & $147 / 123$ & 16.938 & $<0.001$ \\
\hline Maximum diameter $(>/ \leq 7 \mathrm{~cm})$ & $1 / 3 / 157$ & 51.688 & $<0.001$ \\
\hline Total diameter $(>1 \leq 10 \mathrm{~cm})$ & $63 / 207$ & 34.080 & $<0.001$ \\
\hline Tumor number $(>\mid \leq 2)$ & $44 / 226$ & 5.677 & 0.017 \\
\hline $\begin{array}{l}\text { Tumor distribution (single } \\
\text { leaf/bilobar) }\end{array}$ & $232 / 38$ & 0.122 & 0.727 \\
\hline
\end{tabular}

Abbreviations: AFP, alpha-fetoprotein; $\mathrm{HBsAg}$, hepatitis B surface antigen; $\mathrm{HCV}$, hepatitis $\mathrm{C}$ virus; $\mathrm{HT}$, hepatectomy; PFS, progression-free survival; TR, transarterial chemoembolization with radiofrequency ablation.

Table 5 Multivariate analysis of prognostic factors of OS

\begin{tabular}{|l|l|l|l|}
\hline Variable & P-value & RR & $\mathbf{9 5 \% ~ C l ~}$ \\
\hline Treatment (HT/TR group) & 0.007 & 1.562 & $(I .121,2.077)$ \\
$\begin{array}{l}\text { Maximum diameter }(>I \leq 7 \\
\mathrm{cm})\end{array}$ & $<0.00 \mathrm{I}$ & 3.698 & $(2.700,5.064)$ \\
$\begin{array}{l}\text { Tumor number }(>I \leq 2) \\
\text { Antiviral (no/yes) }\end{array}$ & 0.006 & 1.733 & $(I .169,2.570)$ \\
$\begin{array}{l}\text { Child-Pugh classification } \\
\text { (B/A) }\end{array}$ & 0.008 & 0.645 & $(1.046,2.823)$ \\
$\begin{array}{l}\text { Preoperative AFP }(\geq /<400 \\
\mathrm{ng} / \mathrm{mL})\end{array}$ & 0.040 & 1.718 & $(0.467,0.890)$ \\
\hline
\end{tabular}

Abbreviations: AFP, alpha-fetoprotein; HT, hepatectomy; OS, overall survival; RR, risk ratio; TR, transarterial chemoembolization with radiofrequency ablation.
Table 6 Multivariate analysis of prognostic factors of PFS

\begin{tabular}{|l|l|l|l|}
\hline Variable & $\boldsymbol{P}$-value & RR & $\mathbf{9 5 \% ~ C l ~}$ \\
\hline Treatment $(\mathrm{HT} /$ TR group) & $<0.00 \mathrm{I}$ & 2.278 & $(\mathrm{I} .686,3.078)$ \\
$\begin{array}{l}\text { Maximum diameter }(>I \leq 7 \\
\mathrm{cm})\end{array}$ & $<0.00 \mathrm{I}$ & 3.292 & $(2.422,4.475)$ \\
Tumor number $(>I \leq 2)$ & $0.00 \mathrm{I}$ & 1.890 & $(\mathrm{I} .300,2.747)$ \\
\hline
\end{tabular}

Abbreviations: $\mathrm{HT}$, hepatectomy; PFS, progression-free survival; RR, risk ratio; $T R$, transarterial chemoembolization with radiofrequency ablation.

surgical resection. It also causes minimal damage and retains the liver tissue.

It is worth noting that the number of TACE and RFA in patients with recurrence after surgery was similar to that in the TR group. The difference was not statistically significant. However, the OS of the HT group was lower than that of the TR group. It is very interesting! The probable reason was liver function damage and decreased immunity caused by hepatectomy.

The 1-, 2-, 3-, and 5-year OS rates and median OS were $98.5 \%, 83.1 \%, 66.2 \%, 37.1 \%$, and 46 months, respectively, for the TR group and $89.6 \%, 69.4 \%, 53.7 \%, 30.3 \%$, and 38 months, respectively, for the HT group. The OS in the TR group was significantly prolonged compared to that in the HT group. Pan et $\mathrm{al}^{10}$ compared surgical resection with the combination of TACE with RFA for HCC. The median OS and the 1-, 3-, and 5-year survival rates were 61.0 months and $94.4 \%, 83.6 \%$, and $49.5 \%$, respectively, in the resection group and 55.0 months and $95.6 \%, 80.8 \%$, and $40.6 \%$, respectively, in the combination group. Their results of resection group were significantly better than those of the combination group, and their median OS and the 1-, 3-, and 5 -year survival rates of the two groups were higher than those of our corresponding groups. The possible reason was that most patients (60.7\%) enrolled in their study were defined as within the Milan criteria. In addition, their subgroup analysis showed the OS of patients with HCC beyond the Milan criteria in the combination group was significantly prolonged compared to the resection group. This conclusion was the same as in our study. The median OS of the combination group and resection group were 52 and 45 months, respectively, and were higher than corresponding groups. After an in-depth analysis, the reason was found to be that the maximum tumor size beyond the Milan criteria was $\leq 7$ $\mathrm{cm}$ in their study. In our study, the maximum nodule diameter of nearly half of the patients $(41.85 \%)$ was $>7 \mathrm{~cm}$. Yin et al ${ }^{17}$ reported on 88 patients with multiple HCC beyond the Milan criteria who underwent hepatectomy. The 1-, 2-, and 3-year 
OS rates were $76.1 \%, 63.5 \%$, and $51.5 \%$, respectively. Our result was better; the reason might be that they enrolled the patients with at least two nodules.

This study showed that OS, median survival, and PFS of the TR group were longer than those of the HT group. Multivariate analysis also proved that different methods of the groups were important prognostic factors. The combination of TACE and RFA can be complementary and exerts a synergistic therapeutic effect specifically in the following aspects: ${ }^{8}$ 1) TACE can clearly show the tumor contour and increase the rate of complete tumor ablation; 2) TACE can mark and treat lesions that cannot be displayed by enhanced CT/MRI before treatment, and avoid tumor omission during ablation; 3) after TACE, tumor blood supply is significantly reduced; ablation can reduce the heat loss caused by blood flow and increase the rate of complete tumor necrosis; 4) the high thermal energy generated by RFA can effectively enhance the killing of tumor cells by chemotherapeutic drugs; 5 ) by deposition in tumor tissues, the lipiodol improves thermal conduction and enhances the ablation effect; and 6) RFA can reduce the number of TACE treatments and loss of liver function. Studies ${ }^{18,19}$ have confirmed that TACE with RFA resulted in significantly higher tumor necrosis rate than TACE or RFA alone. The combination treatment overcomes the individual limitations of each, is synergistic, and can lead to improved therapeutic outcomes.

The number and diameter of tumors are independent factors that affect OS and are consistent with relevant literatures. ${ }^{20-22}$ The reason is that tumor number and diameter predict the degree of HCC intrahepatic dissemination. Greater the liver tumor burden, greater the size and extent of liver tumor invasion. In addition, longer the tumor diameter, higher the susceptibility to microvascular invasion, and microvascular invasion affects the survival of HCC patients. ${ }^{23}$ Greater the range of liver tissue involved in TACE, RFA, or surgical resection, heavier the damage to liver function, higher the risk of liver failure, and worse the prognosis.

We found in the study that antiviral therapy is an independent factor affecting OS, and HCC is often associated with HBV in China. The risk of postoperative recurrence in HBV-related HCC patients is higher than in HCC patients without HBV. ${ }^{24}$ Treatments such as TACE may activate HBV, causing active HBV-DNA replication, affecting the liver function recovery, leading to liver failure, and promoting recurrence. HBV-DNA affects the prognosis of HBV-related $\mathrm{HCC}$, so antiviral therapy is recommended and can reduce the recurrence rate after $\mathrm{HCC}$ treatment. ${ }^{25,26}$ Therefore, we believe that antiviral drugs should be used before the procedure, and antiviral therapy should be administered throughout the entire HCC treatment process.

The Child-Pugh score predicts liver damage in three different degrees. Survival analysis results showed that the OS time of Child-Pugh A grade HCC patients was significantly better than that of $\mathrm{B}$ grade patients; multivariate Cox regression analysis suggested that Child-Pugh grade was one of the independent factors affecting OS of HCC patients beyond the Milan criteria. The expression level of AFP has a higher predictive value for the prognosis of HCC patients. ${ }^{27}$ For patients with preoperative high expression of AFP, early combination of TACE, sorafenib, and other treatments may help to prolong the survival.

There are limitations to this study. First, it was retrospective with a non-randomized design; there may be an inherent selection bias. Second, it was conducted in a single center. Therefore, the results of this study may be influenced by the experience of the doctor and the patient population. Third, most patients in the TR group had no histological diagnosis. Fourth, the number of patients was relatively small. Finally, most of the patients in this study were patients with HBV-related HCC. A large-scale, multicenter, randomized clinical trial is needed to confirm the findings of our study.

\section{Conclusion}

In conclusion, our study showed that TACE with RFA may lead to better outcomes than hepatectomy in HCC patients beyond the Milan criteria. The treatment method, maximum tumor diameter, and tumor number were independent factors that affected PFS.

\section{Disclosure}

The authors report no conflicts of interest in this work.

\section{References}

1. Jemal A, Bray F, Center MM, Ferlay J, Ward E, Forman D. Global cancer statistics. CA Cancer J Clin. 2011;61(2):69-90.

2. Bosch FX, Ribes J, Borràs J. Epidemiology of primary liver cancer. Semin Liver Dis. 1999;19(3):271-285.

3. El-Serag HB, Rudolph KL. Hepatocellular carcinoma: epidemiology and molecular carcinogenesis. Gastroenterology. 2007;132(7):2557-2576.

4. Parkin DM, Bray F, Ferlay J, Pisani P. Global cancer statistics, 2002. CA Cancer J Clin. 2005;55(2):74-108.

5. Parkin DM. Global cancer statistics in the year 2000. Lancet Oncol. 2001;2(9):533-543.

6. European Association for the Study of the Liver, European Organisation for Research and Treatment of Cancer. EASL-EORTC clinical practice guidelines: management of hepatocellular carcinoma. $J$ Hepatol. 2012;56(4):908-943. 
7. Georgiades CS, Hong K, Geschwind JF. Radiofrequency ablation and chemoembolization for hepatocellular carcinoma. Cancer J. 2008;14(2):117-122.

8. Peng ZW, Zhang YJ, Chen MS, et al. Radiofrequency ablation with or without transcatheter arterial chemoembolization in the treatment of hepatocellular carcinoma: a prospective randomized trial. J Clin Oncol. 2013;31(4):426-432.

9. Yin L, Li H, Li AJ, et al. Partial hepatectomy vs. transcatheter arterial chemoembolization for resectable multiple hepatocellular carcinoma beyond Milan criteria: a RCT. J Hepatol. 2014;61(1):82-88.

10. Pan T, Mu LW, Wu C, et al. Comparison of combined transcatheter arterial chemoembolization and CT-guided radiofrequency ablation with surgical resection in patients with hepatocellular carcinoma within the up-to-seven criteria: a multicenter case-matched study. J Cancer. 2017;8(17):3506-3513.

11. Zhang L, Yin X, Gan YH, et al. Radiofrequency ablation following first-line transarterial chemoembolization for patients with unresectable hepatocellular carcinoma beyond the Milan criteria. BMC Gastroenterol. 2014;14:11.

12. Shi J, Sun Q, Wang Y, et al. Comparison of microwave ablation and surgical resection for treatment of hepatocellular carcinomas conforming to Milan criteria. J Gastroenterol Hepatol. 2014;29(7):1500-1507.

13. Peng ZW, Zhang YJ, Liang HH, Lin XJ, Guo RP, Chen MS. Recurrent hepatocellular carcinoma treated with sequential transcatheter arterial chemoembolization and RF ablation versus RF ablation alone: a prospective randomized trial. Radiology. 2012;262(2):689-700.

14. Lencioni R, Llovet J. Modified RECIST (mRECIST) assessment for hepatocellular carcinoma. Semin Liver Dis. 2010;30(1):052-060.

15. Fernández M, Semela D, Bruix J, Colle I, Pinzani M, Bosch J. Angiogenesis in liver disease. J Hepatol. 2009;50(3):604-620.

16. Liapi E, Geschwind JF. Chemoembolization for primary and metastatic liver cancer. Cancer J. 2010;16(2):156-162.

17. Yin L, Li H, Li AJ, et al. Partial hepatectomy vs. transcatheter arterial chemoembolization for resectable multiple hepatocellular carcinoma beyond Milan criteria: a RCT. J Hepatol. 2014;61(1):82-88.
18. Takaki H, Yamakado K, Uraki J, et al. Radiofrequency ablation combined with chemoembolization for the treatment of hepatocellular carcinomas larger than $5 \mathrm{~cm}$. J Vasc Interv Radiol. 2009;20(2):217-224.

19. Wang ZJ, Wang MQ, Duan F, et al. Clinical application of transcatheter arterial chemoembolization combined with synchronous $\mathrm{C}$-arm cone-beam CT guided radiofrequency ablation in treatment of large hepatocellular carcinoma. Asian Pac J Cancer Prev. 2013;14(3): 1649-1654.

20. Zhao WC, Fan LF, Yang N, Zhang HB, Chen BD, Yang GS. Preoperative predictors of microvascular invasion in multinodular hepatocellular carcinoma. Eur J Surg Oncol. 2013;39(8):858-864.

21. Kim BK, Han KH, Park YN, et al. Prediction of microvascular invasion before curative resection of hepatocellular carcinoma. J Surg Oncol. 2008;97(3):246-252.

22. Hu HT, Kim JH, Lee LS, et al. Chemoembolization for hepatocellular carcinoma: multivariate analysis of predicting factors for tumor response and survival in a 362-patient cohort. J Vasc Interv Radiol. 2011;22(7):917-923.

23. Liu J, Zhu Q, Li Y, et al. Microvascular invasion and positive HB e antigen are associated with poorer survival after hepatectomy of early hepatocellular carcinoma: a retrospective cohort study. Clin Res Hepatol Gastroenterol. 2018;42(4):330-338.

24. Wang Z, Ren Z, Chen Y, et al. Adjuvant transarterial chemoembolization for HBV-related hepatocellular carcinoma after resection: a randomized controlled study. Clin Cancer Res. 2018;24(9):2074-2081.

25. Huang G, Lau WY, Wang ZG, et al. Antiviral therapy improves postoperative survival in patients with hepatocellular carcinoma: a randomized controlled trial. Ann Surg. 2015;261(1):56-66.

26. Chen JL, Lin XJ, Zhou Q, Shi M, Li SP, Lao XM. Association of HBV DNA replication with antiviral treatment outcomes in the patients with early-stage HBV-related hepatocellular carcinoma undergoing curative resection. Chin J Cancer. 2016;35:28.

27. Ma WJ, Wang HY, Teng LS. Correlation analysis of preoperative serum alpha-fetoprotein (AFP) level and prognosis of hepatocellular carcinoma (HCC) after hepatectomy. World J Surg Oncol. 2013;11:212.
Cancer Management and Research

\section{Publish your work in this journal}

Cancer Management and Research is an international, peer-reviewed open access journal focusing on cancer research and the optimal use of preventative and integrated treatment interventions to achieve improved outcomes, enhanced survival and quality of life for the cancer patient. The manuscript management system is completely online and includes

\section{Dovepress}

a very quick and fair peer-review system, which is all easy to use. Visit http://www.dovepress.com/testimonials.php to read real quotes from published authors. 